\title{
Análise da clientela idosa portadora de HIV atendida em um centro ambulatorial em Montes Claros, Minas Gerais
}

\author{
Analysis of elderly HIV clientele attended in an outpatient clinic in Montes Claros city, state of Minas \\ Gerais, Brazil
}

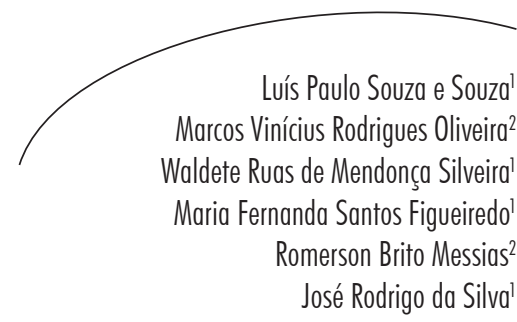

Resumo

A evolução dos casos de infecção pelo HIV em idosos tem sido bastante discutida e chama a atenção pelo impacto que gera na vida dos idosos e na Saúde Pública. Este estudo objetivou conhecer o perfil dos clientes idosos HIV positivo atendidos no Centro Ambulatorial de Especialidades Tancredo Neves (CAETAN), em Montes Claros - MG, Brasil. Estudo documental, quantitativo e descritivo, com coleta de dados secundários. Foram incluídos os prontuários de todos os clientes cadastrados que apresentavam idade igual ou superior a 60 anos; com diagnóstico confirmado de HIV registrado nos impressos de notificação compulsória do Sistema de Informação de Agravos de Notificação (SINAN) e com os prontuários disponíveis no serviço. Foram pesquisados 13 prontuários. Percebeu-se que 76,9\% (n=10) dos idosos eram do sexo masculino e tinham entre 60 e 65 anos de idade; 30,7\% ( $n=07)$ eram casados; 23,1\% ( $n=04)$ cursaram o ensino fundamental; $61,5 \%(n=08)$ eram pardos; $92,3 \%(n=12)$ eram heterossexuais; $46,2 \%(\mathrm{n}=06)$ possuíam parceria única. $53,9 \%(\mathrm{n}=07)$ usavam condom durante relação sexual; $100 \%(n=13)$ se contaminaram por via sexual; $53,9 \%(n=07)$ adquiriram a infecção de profissionais do sexo; 76,9\% $(\mathrm{n}=10)$ faziam uso de antirretrovirais; $53,9 \% \quad(\mathrm{n}=07)$ não possuíam informação no prontuário sobre ter desenvolvido alguma outra doença e se houve apoio da família. Conclui-se que o conhecimento do perfil dos idosos HIV positivos é importante para subsidiar ações de intervenção, que devem abordar aspectos específicos desta população, o que acarretará a quebra do estigma da assexualização dos idosos e em maior conscientização da prevenção para esse grupo.

\section{Abstract}

The evolution of cases of HIV infection in the elderly has been greatly discussed and draws attention by the impact it generates in the life of the elderly and public health. This study aimed to know the profile of HIV-positive elderly in Tancredo Neves Outpatient Center (CAETAN), in Montes Claros city, state of Minas Gerais, Brazil. Documental,

\footnotetext{
Centro de Ciências Biológicas e da Saúde, Departamento de Enfermagem.Universidade Estadual de Montes Claros. Montes Claros, MG, Brasil.

2 Instituto de Ciências da Saúde. Departamento de Medicina. Faculdades Unidas do Norte de Minas.
} Montes Claros, MG, Brasil.

Palavras-chave: Idoso. HIV. Síndrome da Imunodeficiência Adquirida. 
quantitative and descriptive study, with secondary data collection, including medical records of all registered customers aged 60 years or more; diagnosed with HIV with confirmed compulsory notification in the Sistema de Informaşão de Agravos de Notificação (SINAN) and whose medical records were available. Thirteen medical records were searched. It was noticed that $76.9 \%(\mathrm{n}=10)$ elderly were male and were $60-65$ years old; $30.7 \%(\mathrm{n}=07)$ were married; $23.1 \%(\mathrm{n}=04)$ attended elementary school; $61.5 \%(\mathrm{n}=08)$ had brown skin; 92.3\% $(n=12)$ were heterosexuals; $46.2 \%(n=06)$ had unique partnership; $53.9 \%(\mathrm{n}=07)$ wore condom during sexual intercourse; $100 \%(\mathrm{n}=13)$ were sexually infected; $53.9 \%(\mathrm{n}=7)$ acquired the infection from sex workers; $76.9 \%(\mathrm{n}=10)$ made use of antiretroviral drugs; $53.9 \%(\mathrm{n}=07)$ lacked information on record about having developed some other disease and if there was support of the family. It is concluded that the knowledge of elderly HIV-positive is important to subsidize intervention actions, which should address specific aspects of this population, thus breaking the stigma of asexualized elderly and greater awareness of prevention for this group.
Key words: Aged. HIV. Acquired Immunodeficiency Syndrome.

\section{INTRODUÇÃO}

Descoberta em 1981, a síndrome da imunodeficiência adquirida, mais conhecida como Aids, tornou-se um marco histórico da humanidade. A infecção pelo vírus da imunodeficiência humana (HIV) e da Aids tornou-se uma epidemia, representando um fenômeno global, cuja forma de ocorrência nas distintas regiões do mundo depende, entre vários determinantes, do comportamento humano individual e coletivo.

Os dados epidemiológicos desta doença expressam grande significância. Calcula-se que aproximadamente 42 milhões de pessoas no planeta convivem com a Aids e que 2,8 milhões desses infectados apresentam idade superior a 50 anos, compreendendo a população idosa. ${ }^{2}$ No Brasil, de 1980 a junho de 2009, foram diagnosticados 13.665 casos de Aids em pessoas com 60 anos ou mais, dos quais 8.959 em homens e 4.696 em mulheres. ${ }^{3}$

A intensificação dos estudos sobre Aids na população idosa teve seu crescimento, a partir de 2008, com a Campanha do Dia Mundial de Luta Contra a Aids em 2008, com o título "Clube dos Enta", a qual foi voltada para homens heterossexuais com mais de 50 anos pertencentes às classes $\mathrm{C}$ e $\mathrm{D}$. A escolha desse público se deu, principalmente, porque a incidência de Aids praticamente dobrou nessa população nos anos de 1996 a 2006: de 7,5\% para 15,7\% ${ }^{4}$
Desde o início, em relação à infecção pela Aids, usava-se o termo "grupo de risco" para delimitar ou direcionar pessoas, de forma preconceituosa, apenas pelo fato de se enquadrarem em certos grupos populacionais por exemplo, homossexuais e prostitutas -, por desenvolverem certos hábitos considerados de risco para tal agravo. Entretanto percebe-se que, mais recentemente, esse termo cedeu lugar ao intitulado "comportamento de risco".

A associação da doença aos grupos de risco dissemina a falsa noção de que as pessoas não pertencentes a esses "grupos" estariam a salvo da ameaça. A noção de comportamento de risco considera as práticas que levam o indivíduo a um menor ou maior grau de exposição ao HIV. Assim, os idosos são bastante vulneráveis e fazem parte dos grupos que possuem comportamento de risco, devido ao aumento da prática sexual, principalmente masculino, em decorrência do advento de drogas estimuladoras e a resistência ou deficiência de orientações com relação ao uso do condom. ${ }^{5}$

Por se considerarem um grupo com menos riscos de contrair a Aids, as pessoas com idade mais avançada costumam adiar a realização do teste anti-HIV. No início da epidemia da Aids, os idosos se contaminavam por meio de transfusões sanguíneas. Entretanto, a transmissão por essa rota diminuiu sistematicamente com a iniciação de exames médicos solicitados a doadores de sangue. Com o passar dos anos, a infecção do HIV em pessoas com maior idade foi sendo frequentemente transmitida pela via sexual. ${ }^{6}$ 
Na mídia, são diversas as propagandas que prometem acabar com a impotência sexual; no entanto, as campanhas de prevenção de transmissão do HIV são mais direcionadas aos adolescentes e jovens, sendo escassas para a população idosa. Assim, o crescimento do número de infecções por HIV em pessoas com 60 anos ou mais resulta na mais nova característica da epidemia. ${ }^{7}$

Dois fatores podem estar relacionados aos crescentes números de casos de Aids em idades mais avançadas. $O$ primeiro se deve àqueles idosos que possuem, entre outros fatores, mais recursos, o que contribui para o acesso aos prazeres e serviços disponíveis, permitindo vida sexual mais ativa. A existência de tabu sobre a sexualidade na terceira idade é o segundo fator. É enganoso pensar que as pessoas idosas não possuem atividade sexual e não fazem uso de drogas, assim, de modo geral, estas pessoas estão menos informadas sobre o HIV e pouco conscientes de como se protegerem. É um mito pensar em velhice sem sexualidade.

Em virtude da estigmatização da terceira idade, tanto os familiares como os profissionais negam-se a pensar que nessa fase a pessoa está ativa sexualmente. Essa falha traz graves consequências, sobretudo quanto à prevenção, pois esta só vai ocorrer se os familiares e profissionais de saúde estiverem atentos para discutir abertamente as formas de prevenção. Para conter essa perspectiva de epidemia, é necessário quebrar o tabu social relacionado ao sexo entre as pessoas mais experientes, inclusive abolir a ideia de assexualização, sobretudo entre os profissionais de saúde. ${ }^{8}$

Diante da perspectiva do aumento do número de idosos infectados na terceira idade, pela descoberta de novos casos nesta faixa etária e em decorrência da ampliação da expectativa de vida dos indivíduos com Aids, facilitada pelos antirretrovirais, faz-se necessário conhecer as características regionais dessa população já acometida. ${ }^{6,8}$ Torna-se imprescindível a discussão das questões sobre a sexualidade na terceira idade, e conhecer sua realidade. Constituem-se um desafio para a saúde pública.
Em face do exposto, elaborou-se este estudo, cujo objetivo foi conhecer o perfil dos clientes idosos HIV positivo atendidos no Centro Ambulatorial de Especialidades Tancredo Neves da Universidade Estadual de Montes Claros, da cidade de Montes Claros, Estado de Minas Gerais.

\section{METODOLOGIA}

Realizou-se estudo documental, quantitativo e descritivo, com coleta de dados secundários, a partir de prontuários dos idosos atendidos pelo Centro Ambulatorial de Especialidades Tancredo Neves (CAETAN), localizado em Montes Claros, Minas Gerais, Brasil.

Definiu-se esse centro como cenário da pesquisa pelo fato de ser este o local de referência para atendimento de clientes HIV-positivo de Montes Claros, dos municípios do norte de Minas Gerais e sul da Bahia, oferecendo atendimento clínico especializado a esses pacientes. $\mathrm{O}$ CAETAN faz parte da estrutura organizacional da Universidade Estadual de Montes Claros (Unimontes) e do Hospital Universitário Clemente de Faria (HUCF).

No período do estudo, o CAETAN apresentava 253 prontuários cadastrados. Foram incluídos no estudo os prontuários de todos os clientes cadastrados no CAETAN, que apresentavam idade igual ou superior a 60 anos; com diagnóstico confirmado de HIV registrado nos impressos de notificação compulsória do Sistema de Notificação de Informação de Agravos de Notificação (SINAN) e com os prontuários disponíveis no serviço. Assim, dos 15 prontuários de idosos com confirmação ou suspeita de HIV que o Centro Ambulatorial de Especialidades Tancredo Neves dispunha, 13 foram incluídos neste estudo, uma vez que em um dos prontuários o paciente estava ainda em processo de investigação da infecção pelo HIV e um prontuário não foi encontrado no serviço.

A coleta de dados foi realizada no segundo semestre de 2007, utilizando como instrumento um formulário abordando as variáveis "sexo", "faixa etária", "escolaridade", "raça", "estado 
civil", "comportamento sexual", "via de contágio", "número de parceiros sexuais", "fonte de infecção", "uso do preservativo", "uso de antirretrovirais", ser "portador de outra patologia crônica" e ter "apoio da família".

Com os dados coletados, elaborou-se um banco de dados utilizando o programa Microsoft Excel 2003, que, posteriormente, com auxílio do programa Epi-Info versão 3.5, permitiu analisar e interpretar as variáveis em questão, por meio da análise descritiva dos dados.

Os aspectos éticos desta pesquisa foram considerados de acordo com a Resolução $\mathrm{n}^{\circ}$ 196/96 da Comissão Nacional de Ética do Ministério da Saúde, que estipula normas éticas regulamentadoras de pesquisas envolvendo seres humanos. O presente estudo foi aprovado pelo Comitê de Ética em Pesquisa da Universidade Estadual de Montes Claros, por meio do parecer consubstanciado número 743/07.

\section{RESULTADOS}

Foram pesquisados 13 prontuários. No que diz respeito aos dados socioeconômicos, percebeu-se que $76,9 \%(n=10)$ dos idosos eram do sexo masculino; $76,9 \%(\mathrm{n}=10)$ encontravamse entre a idade de 60 e 65 anos; 30,7\% ( $\mathrm{n}=07)$ eram casados; $23,1 \%(n=04)$ cursaram o ensino fundamental; e $61,5 \%(\mathrm{n}=08)$ eram pardos; como evidenciado na tabela 1.

Tabela 1 - Perfil socioeconômico dos idosos portadores de HIV/Aids cadastrados no Centro Ambulatorial de Especialidades Tancredo Neves, Montes Claros - MG, outubro de 2007.

\begin{tabular}{lllc}
\hline Variáveis & & $\mathrm{N}$ & $\%$ \\
\hline Sexo & Masculino & 10 & 76,9 \\
& Feminino & 03 & 23,1 \\
Idade (anos) & $60-65$ & 10 & 76,9 \\
& $66-70$ & 01 & 7,7 \\
& Maior que 71 & 02 & 15,4 \\
& & & \\
Estado civil & Solteiro & 04 & 30,7 \\
& Casado/União Consensual & 07 & 53,9 \\
& Separado/Divorciado & 01 & 7,7 \\
& Viúvo & 01 & 7,7 \\
& Analfabeto & & 15,4 \\
Escolaridade & Fundamental & 02 & 30,7 \\
& Ensino médio & 04 & 7,7 \\
& Superior & 01 & 23,1 \\
& & 03 & 23,1 \\
Raça & Não informado & 03 & 00 \\
& Negro & 00 & 38,5 \\
& Branco & 05 & 61,5 \\
\hline
\end{tabular}

Fonte: Fichas individuais e prontuários de atendimento clínico dos clientes cadastrados no CAETAN, outubro de 2007. 
Quanto aos dados sobre a sexualidade, os achados foram agrupados na tabela 2 . Verificouse que $92,3 \%(n=12)$ eram heterossexuais; $46,2 \%$
( $\mathrm{n}=06)$ possuíam parceria única; e 53,9\% ( $\mathrm{n}=07$ ) usavam condom durante relação sexual.

Tabela 2 - Caracterização dos idosos portadores de HIV/Aids cadastrados no Centro Ambulatorial de Especialidades Tancredo Neves quanto à sexualidade. Montes Claros - MG, outubro de 2007.

\begin{tabular}{llcc}
\hline Variáveis & & $\mathrm{N}$ & $\%$ \\
\hline Comportamento sexual & Homossexual & 01 & 7,7 \\
& Bissexual & 00 & 0,0 \\
& Heterossexual & 12 & 92,3 \\
& & & \\
Parceria sexual & Única & 06 & 46,2 \\
& Múltipla & 04 & 30,7 \\
& Não tem & 01 & 7,7 \\
& Não informado & 02 & 15,4 \\
& & & 7,7 \\
Uso de preservativos & Sempre & 01 & 7,7 \\
& Às vezes & 01 & 53,9 \\
& Nunca & 07 & 30,7 \\
\hline
\end{tabular}

Fonte: Fichas individuais e prontuários de atendimento clínico dos clientes cadastrados no SAE do CAETAN, outubro de 2007.

Outros aspectos analisados na presente pesquisa foram as vias e a forma de infecção pela doença, conforme mostra a tabela 3. Nota-se que a única via de contágio relatada foi a sexual. Em se tratando da fonte de infecção, a tabela 3 mostra que $53,8 \%(\mathrm{n}=07)$ dos idosos $(53,8 \%)$ adquiriram a infecção de profissionais do sexo.

Tabela 3 - Via de contágio e fontes de infecção entre os idosos portadores de HIV/Aids cadastrados no Centro Ambulatorial de Especialidades Tancredo Neves. Montes Claros - MG, outubro de 2007.

\begin{tabular}{llcc}
\hline Variáveis & & $\mathrm{N}$ & $\%$ \\
\hline Via de contágio & Sexual & 13 & 100,0 \\
& Sanguínea (transfusão) & 00 & 0,0 \\
& Acidente de trabalho & 00 & 0,0 \\
& & & \\
Fonte de infecção & Cônjuge & 03 & 23,1 \\
& Profissionais do sexo & 07 & 53,8 \\
& Transfusão de hemoderivados & 00 & 0,0 \\
& Não informado & 03 & 23,1 \\
\hline
\end{tabular}

Fonte: Fichas individuais e prontuários de atendimento clínico dos clientes cadastrados no SAE do CAETAN, outubro de 2007. 
A tabela 4 mostra a distribuição por uso de antirretrovirais, acometimento de outras doenças e apoio da família após instalação do HIV. Notou-se que 76,9\% (n=10) dos idosos faziam uso de antirretrovirais; 53,9\% $(\mathrm{n}=07)$ não possuíam informação no prontuário sobre ter desenvolvido ou não alguma outra doença; e 53,9\% (n=07) não possuíam informação no prontuário se houve ou não apoio da família.

Tabela 4 - Distribuição dos idosos portadores de HIV/Aids cadastrados no Centro Ambulatorial de Especialidades Tancredo Neves por uso de antirretrovirais, acometimento de outras doenças e apoio da família após instalação do HIV. Montes Claros -MG, outubro de 2007.

\begin{tabular}{llcc}
\hline Variáveis & & $\mathrm{N}$ & $\%$ \\
\hline Uso de antirretroviral & Sim & 10 & 76,9 \\
& Não & 01 & 7,7 \\
& & 02 & 15,4 \\
Dõo informado & & \\
& Diabetes crônicas & 02 & 15,4 \\
& Hipertensão & 04 & 30,7 \\
Câncer de próstata & 00 & 0,0 \\
& Câncer de útero & 00 & 0,0 \\
Não informado & 07 & 53,9 \\
& Sim & 04 & 30,7 \\
Apoio da família & Não & 02 & 15,4 \\
& Não informado & 07 & 53,9 \\
\hline
\end{tabular}

Fonte: Fichas individuais e prontuários de atendimento clínico dos clientes cadastrados no SAE do CAETAN, outubro de 2007.

\section{DISCUSSÃO}

O primeiro caso de Aids notificado em pessoas acima de 50 anos de idade foi em 1982. Desde então, até o período de junho de 2008, em todo o mundo, identificaram-se 47.437 casos, sendo 15.966 (34\%) entre mulheres e 31.468 entre homens. ${ }^{3}$ Em estudo realizado com 20 idosos com HIV confirmados nas fichas de notificação do SINAN, no Complexo Hospitalar Clementino Fraga, em João Pessoa, Estado da Paraíba, encontrou-se que a maioria eram homens $(55 \%){ }^{9}$ Maior prevalência de HIV no sexo masculino também foi observada no presente estudo.
Quanto à idade e ao estado civil, os dados mostraram que a maior parte dos idosos encontrava-se entre 60-65 anos de idade e era casada. Tais achados são semelhantes ao estudo feito por Sousa, Suassuna e Costa, ${ }^{9}$ que observaram que a faixa etária de maior incidência foi $59-65$ anos $(50 \%)$ e $67-69(50 \%)$.

Com a análise dos prontuários neste estudo, constatou-se que a maior parte dos idosos com HIV pesquisados era casada. Corroborando, autores verificaram em estudo que dos 22 idosos com HIV analisados, metade era casada ou possuía relação estável. ${ }^{10}$ Torna-se importante discutir e 
incentivar a população sobre o uso de condom, como forma de prevenção, mesmo em relações consideradas "seguras" e "estáveis". ${ }^{11}$ Ressalta-se que, entre os idosos que se descobrem portadores do vírus, há dois perfis clássicos: o homem casado que se contamina com uma parceira mais jovem e o das viúvas que redescobrem o sexo. ${ }^{12}$ Gir et al. ${ }^{13}$ esclarecem que o estado civil não é um indicador confiável para avaliação da atividade sexual ou do tipo de parceria.

A maior parte dos idosos deste estudo possuía escolaridade até o ensino fundamental, seguido por nível superior e analfabeto, assemelhando-se ao encontrado no estudo de Bertoncini, Moraes \& Kulkamp, ${ }^{10}$ em que dos 12 entrevistados, $54,5 \%$ apresentaram primeiro grau incompleto e $13,6 \%$ possuíam curso superior. Andrade, Silva \& Santos ${ }^{14}$ também encontraram resultados semelhantes no estudo com 13 idosos soropositivos, tendo evidenciado predominância da $5^{\mathrm{a}}$ série primária, correspondendo hoje à $5^{a}$ série do ensino fundamental, como nível de escolaridade. Segundo dados do Boletim Epidemiológico da Aids e Doenças Sexualmente Transmissíveis do Ministério da Saúde do Brasil, divulgados no ano de 2008, tem-se percebido um aumento no número de casos de HIV em pessoas com baixa escolaridade, com média de estudo entre quatro a sete anos. ${ }^{3}$ Tal fato remete a uma reflexão a respeito das estratégias das campanhas educativas, que precisam ser claras, considerando-se o nível de compreensão de pessoas com menos instrução formal. ${ }^{15}$

Em se tratando da cor de pele, os prontuários evidenciaram que a maior parte dos idosos analisados eram pardos. Já em outro estudo, entre os idosos soropositivos, $62,8 \%$ eram da cor branca. ${ }^{16}$

No que diz respeito à forma de infecção pelo HIV, os dados evidenciaram que todos os idosos contraíram o vírus por meio de relação sexual. Corroborando tais achados, Bertoncini et al. ${ }^{11}$ avaliaram pessoas com mais de 50 anos de idade soropositivas e observaram que a relação sexual foi a forma de contágio do HIV mais predominante $(72,8 \%)$. Lisboa ${ }^{17}$ destaca que a principal causa da contaminação por HIV em pessoas acima de 50 anos remete à atividade sexual.

Dessa forma, o aumento das práticas sexuais entre os indivíduos da terceira idade deve estar associado às iniciativas de prevenção e de assistência por parte dos profissionais da saúde, para um controle mais preciso dos eventos relacionados com a exposição desses indivíduos a Aids e demais doenças sexualmente transmissíveis. Os idosos soropositivos necessitam de maior atenção à saúde, pois, pela idade avançada, têm demandas específicas que devem ser consideradas. Além disso, é de fundamental importância para esses idosos compreender sua sexualidade e os meios de proteção para práticas sexuais seguras. ${ }^{9}$

Como fonte de infecção, a maior parte dos idosos deste estudo adquiriu a doença por meio de profissionais do sexo. Os idosos que possuem, entre outros fatores, maior poder aquisitivo e outros recursos, têm mais condições de buscar prazeres e serviços disponíveis, tendo uma vida sexual mais ativa, aumentando assim os riscos de contrair doenças sexualmente transmissíveis. ${ }^{18}$ As aceitações sociais com múltiplas parceiras permanecem na trajetória da vida dos homens que hoje têm mais de 60 anos, os quais não praticam sexo seguro, porque isso possivelmente nunca fez parte da vida deles, o que resulta da construção social e de gênero. ${ }^{19}$

No Brasil, os primeiros casos de Aids registrados foram, na maior parte, em homossexuais masculinos. No período de 1990 a junho de 2008, notou-se aumento da transmissão heterossexual masculina, com estabilização no final do período. $\mathrm{Na}$ subcategoria homossexual/bissexual, notouse diminuição da proporção de casos, também seguida de estabilização no final do período. Em mulheres idosas, há o predomínio de casos de transmissão heterossexual em todo o período. ${ }^{3}$

O perfil epidemiológico da população infectada pelo HIV modificou-se nos últimos 
anos, atingindo também heterossexuais, ${ }^{20}$ como verificado neste estudo, em que a maioria era heterossexual, seguidos de apenas um idoso homossexual. À semelhança desses resultados, Bertoncini, Moraes \& Kulkamp ${ }^{11}$ observaram que, dos idosos com Aids pesquisados, $63,6 \%$ eram heterossexuais, $31,8 \%$ dos casos homossexuais e $4,5 \%$ transexuais. Autores afirmam que a heterossexualidade é a categoria que mais contribui com o avanço da epidemia. ${ }^{21}$

Quanto à parceria sexual, a maior parte dos sujeitos possuía parceiro único. No entanto, estudo ressalta que ainda perdura a multiplicidade de parceiros, na trajetória da vida dos indivíduos, mas especificamente entre os homens. ${ }^{19}$

Em relação ao uso de condom, os prontuários evidenciaram que a maioria dos idosos pesquisados não o utilizava nas relações sexuais. Outros estudos mostraram comportamento semelhante., ${ }^{922}$ Caldas \& Gessolo ${ }^{22}$ observaram que a população masculina acima de 50 anos de idade deixou de usar o condom em virtude do medo de comprometer a ereção peniana, utilizando-o apenas em relações extraconjugais.

A importância do uso de preservativos deve ser sempre destacada, mesmo se ambos os parceiros estejam infectados pelo HIV, uma vez que o não-uso pode propiciar a infecção por outras doenças sexualmente transmissíveis (DSTs), dificultando o controle da carga viral, o que pode aumentar o risco de contaminação por vírus resistentes aos antirretrovirais. ${ }^{21,23}$

Por medo de interferir na vida sexual e afetiva do casal, muitos idosos optam em não utilizar o preservativo. Autores perceberam a resistência masculina quanto a seu uso, independentemente de a parceira ser soropositiva ou não, o que constitui importante aspecto de exposição das parceiras soronegativas. Destaca-se, ainda, que o uso do preservativo após a descoberta da infecção pode acarretar modificações e mudanças de hábitos que nem sempre são fáceis. ${ }^{24}$

A presente pesquisa verificou que $76,9 \%$ dos idosos utilizam os antirretrovirais, o que pode ser considerado um fator positivo, tendo em vista as inúmeras dificuldades encontradas pelos clientes, dentre elas a maior sensibilidade aos efeitos colaterais e a possível associação com outras drogas utilizadas. A terapia antirretroviral, além de proporcionar maior sobrevida, contribui para que os idosos portadores do HIV continuem suas atividades cotidianas, o que levanta questões a respeito da importância das redes de apoio social e da participação social como fatores relevantes no enfrentamento da soropositividade ao HIV. ${ }^{25,26}$

Devido ao envelhecimento e pela presença de doenças metabólicas, há maior dificuldade de tratar este grupo etário, o que compromete a escolha da terapêutica adequada (antirretrovirais), por conta dos efeitos colaterais, que agravariam as alterações preexistentes. Alia-se o fato de muitos pacientes terem seu diagnóstico estabelecido tardiamente, apresentando com doenças oportunistas que elevam a dificuldade de controle da Aids. ${ }^{27}$

Outro fator preponderante na infecção HIV nos idosos relaciona-se com outras doenças crônicas, as quais mascaram os sintomas da Aids e podem induzir um diagnóstico errado ou, de certo modo, inconclusivo. Neste estudo, verificou-se que a maior parte dos idosos possuía hipertensão, seguida de diabetes. Ressalta-se que estas enfermidades são comuns na terceira idade, tendendo a indivíduos de idade mais avançada. ${ }^{28-}$

Considerando o enfrentamento da enfermidade ou da situação de soropositivo, algumas distinções podem ser observadas, caso o indivíduo envelheça contaminado pelo HIV ou adquira a infecção ou a doença propriamente dita em uma fase mais avançada de sua vida. O idoso tem uma tendência natural quando se depara com a situação de adoecer devido à Aids, de ter mais medo da morte, mas tudo isso depende de toda uma complexidade psicológica, familiar e social. Em situação de apoio, melhorias são observadas na saúde do idoso, sua adesão ao tratamento é melhor, e ele passa a ter mais cuidados com alimentação, exercícios e medicação, resultando num sucesso terapêutico maior. ${ }^{16}$ No presente estudo, não se sabe se o apoio familiar poderia 
ser um fator protetor, uma vez que esta informação não estava presente na maior parte dos prontuários.

A crença de que os idosos não estão susceptíveis a contraírem HIV ou outras doenças sexualmente transmissíveis é um dos desafios da prevenção do HIV e deve ser desmistificado. Torna-se imprescindível conscientizar os profissionais de saúde sobre seu papel na prevenção da doença e a realização de atividades de educação em saúde para os idosos, a respeito dos riscos e formas de prevenção do HIV. ${ }^{24,29}$

\section{CONCLUSÃO}

Este estudo tem como limitação o pequeno número de prontuários pesquisados, o que limitou inferências gerais à população. Além disso, a dificuldade de se encontrar o registro de notificação compulsória do Sistema de Informação de Agravos de Notificação (SINAN) nos prontuários e a qualidade das informações prestadas tornaram-se fatores limitantes para a coleta de dados.

A análise dos prontuários demonstrou que a maior parte dos idosos portadores de HIV cadastrados no Centro Ambulatorial de Especialidades Tancredo Neves (CAETAN) era do sexo masculino, encontrava-se na faixa etária entre 60 e 65 anos de idade, possuía o ensino fundamental, sendo parda e casada. Quanto ao comportamento sexual, a maioria era formada por heterossexuais, adquiriu a infecção por via sexual, possuía parceria sexual única, mas adquiriu a infecção por meio de relações sexuais com profissionais do sexo e nunca utilizava o preservativo. Com relação ao tratamento, a maioria utilizava medicamentos antirretrovirais e possuía outras patologias crônicas.

Ressalta-se a necessidade da realização de ações de prevenção, que devem ser realizadas em todos os serviços de saúde, de forma a sensibilizar os profissionais de saúde sobre esse problema e a necessidade de implementar estratégias que mudem essa realidade.

Enfatiza-se a importância de os profissionais de saúde acolherem o idoso portador de HIV sem discriminação, independentemente de sua condição, escolaridade, orientação sexual ou estilo de vida, de forma integral e humanizada, por uma equipe multiprofissional.

As atividades educativas sobre o HIV devem abordar também os aspectos específicos dessa faixa etária. Isso acarretará na quebra do estigma da assexualização de pessoas idosas, com maior conscientização sobre a prevenção para esse grupo.

Por fim, é preciso entender que o envelhecimento significa um processo que reflete uma vida inteira de hábitos, crenças, experiências e ensinamentos, os quais devem ser respeitados e levados em consideração por toda a população.

\section{REFERÊNCIAS}

1. Brasil. Ministério da Saúde. Programa Nacional de DST/AIDS. A epidemia de AIDS no Brasil. Bol Epidemiol DST/AIDS [Internet]. 2009 [Acesso em 2012 jul 14]; 6(1). Disponível em: http://www. aids.gov.br/sites/default/files/publicacao/2009/ boletim2009_final_pdf_24513.pdf.

2. Nguyen N, Holodniy M. HIV infection in the elderly. Clinical Interventions in Aging 2008; 3(3): 453-472.

3. Ministério da Saúde (BR). Programa Nacional de DST/AIDS. Municípios com pelo menos um caso de aids em indivíduos com 50 anos ou mais. Bol Epidemiol DST/AIDS 2008; 5(1)

4. Ministério da Saúde (BR). Secretaria de Atenção à Saúde. Departamento de Atenção Básica. Caderno de Atenção Básica: envelhecimento e saúde da pessoa idosa. Brasília: DF; 2006.

5. Gomes SF, Silva CM. Perfil dos idosos infectados pelo HIV/AIDS: uma revisão. Vittalle 2008; 20(1): 107-122.

6. Araújo VLB, et al. Características da Aids na terceira idade em um hospital de referência do Estado do Ceará, Brasil. Rev Bras Epidemiol 2007; 10(4): 544-54.

7. Sousa JL. Sexualidade na terceira idade: uma discussão da AIDS, envelhecimento e medicamentos para disfunção erétil. DST J Bras Doenças Sex Transm 2008; 20(1):59-64. 
8. Maschio MBM, Balbino AP, Souza PFR, Kalinke LP. Sexualidade na terceira idade: medidas de prevenção para doenças sexualmente transmissíveis e AIDS. Rev Gaúcha Enferm 2011 Set [Acesso em 2012 Jul 14]; 32(3): 583-589.

9. Sousa ACA, Suassuna DSB, Costa SML. Perfil Clínico-Epidemiológico de Idosos com aids. DST - J bras Doenças Sex Transm 2009: 21(1): 22-26.

10. Bertoncini BZ, Moraes KS, Kulkamp IC. Comportamento sexual em adultos maiores de 50 anos infectados elo HIV. DST - J bras Doenças Sex Transm 2007; 19(2): 75-79.

11. Gross, JB. Estudo de pacientes portadores de HIV/ AIDS após os 60 anos de idade em duas unidades de saúde do estado do Rio de Janeiro. [dissertação] Rio de Janeiro: Escola Nacional de Saúde Pública, Fundação Oswaldo Cruz; 2005.

12. Fontes KS, Saldanha AAW, Araujo LF. Representações do HIV na terceira idade e a vulnerabilidade no idoso. VII Congresso Virtual HIV/AIDS. Portugal; 2006 -[Acesso em 2012 jul 13]. Disponível em: http://www.aidscongress. net/Modules/WebC_Docs/GetDocument. aspx?DocumentId=241

13. Gir E, et al. A parceria sexual na visão de mulheres portadoras do vírus da imunodeficiência humana-HIV. DST - J bras Doenças Sex Transm 2006; 18(1): 53-57.

14. Andrade HAS, Silva SK, Santos MIPO. Aids em idosos: vivências dos doentes. Esc Anna Nery (impr.) 2010 out-dez; 14(4):712-719.

15. Fonseca MG, et al. AIDS e grau de escolaridade no Brasil: evolução temporal de 1986 a 1996. Cad Saúde Pública 2000; 16(Sup. 1): 77-87.

16. Lopes PSD, Silva MMG, Torres IC, Stadñik CMB. Rev AMRIGS 2011; 55(4): 356-360.

17. Lisboa MES. A invisibilidade da população acima de 50 anos no contexto da epidemia HIV/AIDS. V Congresso Virtual HIV/AIDS.Portugal; 2004 - [ Acesso em 03 de janeiro de 2011]. Disponível em: http://www.aidscongress.net/article.php?id_ comunicacao $=281$.

18. Brasil. Ministério da Saúde. Cresce o número de portadores de AIDS na terceira idade. Jornal a Tribuna Digital. Santos, SP; 2004. [Acesso em 2012 jul 13]. Disponível em: http://www.AIDS.gov.br/ imprensa/noticias

19. Saldanha AAW, Araújo LF, Sousa VC. Envelhecer com Aids: representações, crenças e atitudes de idosos soropositivos para o HIV. Rev Interam Psicol 2009; 43(2): 323-332.

20. Szwarcwald CL, Bastos FI, Esteves MAP, Andrade CLT. A disseminação da epidemia da AIDS no Brasil, no período de 987-1996: uma análise espacial. Cad Saúde Pública 2000; 16 (1): 7-19.

21. Reis RK, Gir E. Dificuldades enfrentadas pelos parceiros sorodiscordantes ao HIV na manutenção do sexo seguro. Rev Latino-am Enfermagem 2005; 13(1): 327.

22. Caldas JMP, Gessolo KM. AIDS depois dos 50: um novo desafio para as políticas de saúde pública. V Congresso Virtual HIV/AIDS.Portugal; 2004 - [ Acesso em 14 de abril de 2011]. Disponível em: http://www.aidscongress.net/article.php?id_ comunicacao $=285$.

23. Mattos SAF, Assis M. Vulnerabilidade das idosas ao HIV/AIDS: despertar das Políticas Públicas e profissionais de saúde no contexto da atenção integral: revisão de literatura. Rev Bras Geriatr Gerontol. 2011. [Acesso em 2012 jul 13]; 14(1): 147-158. Disponível em: <http://revista.unati.uerj. br/scielo.php?script $=$ sci_arttext\&pid=S180998232011000100015\&lng=pt $>$.

24. Silva HR, Marreiros MDÓC, Figueiredo TS, Figueiredo MLF. Características clínicoepidemiológicas de pacientes idosos com aids em hospital de referência, Teresina-PI, 1996 a 2009. Epidemiol Serv Saúde. 2011; 20(4): 499-507.

25. Araújo CLO, Moura LF, Cardoso NA. Caracterização do portador de HIV/AIDS acima de 50 anos. Kairós Geront 2009 nov; 12(2): 173-82.

26. Costa DA, Zago MMF, Medeiros M. Experiência da adesão ao tratamento entre mulheres com vírus da imunodeficiência humana/síndrome da imunodeficiência humana. Acta Paul Enferm 2009 mar; 22(5): 631-37.

27. Vieira DLFC, Sobral B. O corpo envelhece, a sexualidade não: AIDS no diagnóstico diferencial entre as doenças comuns nos idosos. Int Clin Med 2009 [Acesso em 2012 jul 12]; 12(B6). Disponível em: http://www.medcenter.com/medscape/content. aspx?id=18356\&lang'Type $=1046$

28. Araújo GBS, Garcia TR. Adesão ao tratamento antihipertensivo: uma análise conceitual. Rev Eletrônica Enferm 2006; 8(2): 259- 272.

29. Melo HMA, Leal MCC, Marques APO, Marino JG. O conhecimento sobre Aids de homens idosos e adultos jovens: um estudo sobre a percepção desta doença. Ciên Saúde Coletiva 2012; 17(1): 43-53. 